\title{
O poder da universalidade: o acesso à saúde dos migrantes na atenção primária à saúde no Vale do Paranhana/RS, uma concepção dos profissionais de saúde
}

\section{The power of universality: The access to health of migrants in Primary Health Care in Valley of Paranhana/RS, a conception of health professionals}

\author{
Samanta Andresa Richter ${ }^{1 *}$, Maisson da Silva Berg ${ }^{2}$, Daniel Luciano Gevehr ${ }^{2}$
}

\section{RESUMO}

O estudo teve como objetivo analisar as concepções dos profissionais da saúde acerca do acesso à saúde prestado à população migrante. Trata-se de um estudo qualitativo, exploratório-descritivo. Para a realização do estudo, foram entrevistados 7 enfermeiros e 2 Agentes Comunitários de Saúde (ACSs). O período de coleta de dados foi nos meses de outubro a novembro de 2019. Para análise dos dados foi utilizada a análise de conteúdo, proposta por Minayo (2014). Este estudo respeita os aspectos bioéticos referente às pesquisas com seres humanos. Para elucidar os resultados emergiram três categorias temáticas, a saber: "como cheguei aqui?" o processo de inserção e acesso à saúde dos migrantes; barreiras para o atendimento prestado aos migrantes residentes; e significados que permeiam os profissionais de saúde no atendimento ao migrante, as quais elucidam os principais aspectos encontrados pelas entrevistadas dos profissionais. Um dos principais aspectos que os profissionais relataram com relação às barreiras, foram o idioma e a comunicação. Contudo, ressalta-se que o estabelecimento do vínculo entre usuário - profissional de saúde - serviço de saúde, podem fortalecer o acesso à saúde da população de migrantes.

Palavras-chave: Saúde do migrante; Acesso à saúde; SUS; Políticas públicas de saúde.

\section{ABSTRACT}

The study aimed to analyze the conceptions of health professionals about the access to health provided to the migrant population. This is a qualitative, exploratory-descriptive study. To carry out the study, 7 nurses and 2 Community Health Agents (CHAs) were interviewed. The data collection period was from October to November 2019. For data analysis, the content analysis proposed by Minayo (2014) was used. This study respects the bioethical aspects related to research with human beings. To elucidate the results, three thematic categories emerged, namely: "how did I get here?" the process of insertion and access to health care for migrants; barriers to the care provided to resident migrants; and meanings that permeate health professionals in assisting migrants, which elucidate the main aspects found by the professionals' interviewees. One of the main aspects that professionals reported regarding barriers were language and

1 Programa de Pós-graduação em Pediatria e Saúde da Criança (PPGPSC). Pontifícia Universidade Católica do Rio Grande do Sul (PUCRS). *E-mail: SAMANTA.RICHTER@edu.pucrs.br

${ }^{2}$ Programa de Pós-graduação em Desenvolvimento Regional (PPGDR). Faculdades Integradas de Taquara (FACCAT). 
communication. However, it is noteworthy that the establishment of the link between user - health professional - health service can strengthen the access to health of the migrant population.

Keywords: Migrant health; Access to health; SUS; Public health policies.

\section{INTRODUÇÃO}

O Sistema Único de Saúde (SUS) é um dos maiores e mais complexos sistemas de saúde pública do mundo, abrangendo desde o simples atendimento para avaliação da pressão arterial, por meio da Atenção Primária à Saúde (APS), até o transplante de órgãos, garantindo acesso integral, universal e gratuito para toda a população do país (BRASIL, 2019). Com a sua criação, o SUS proporcionou o acesso universal ao sistema público de saúde, sem discriminação. A atenção integral à saúde, e não somente aos cuidados assistenciais, passou a ser um direito de todos os brasileiros, desde a gestação e por toda a vida, com foco na saúde com qualidade de vida, visando a prevenção e a promoção da saúde (BRASIL, 2019).

Com isso, a nova Política Nacional de Atenção Básica (PNAB), em 2017, aborda que a APS é o conjunto de ações de saúde individuais, familiares e coletivas que envolvem promoção, prevenção, proteção, diagnóstico, tratamento, reabilitação, redução de danos, cuidados paliativos e vigilância em saúde, desenvolvida por meio de práticas de cuidado integrado e gestão qualificada, realizada com equipe multiprofissional e dirigida à população em território definido, sobre as quais as equipes assumem responsabilidade sanitária. Ou seja, a APS deve ser incorporada como a principal porta de entrada ao acesso à saúde e centro de comunicação da Rede de Atenção à Saúde (RAS), coordenadora do cuidado e ordenadora das ações e serviços disponibilizados na rede (MENDES, 2011).

Dentro do que se compreende as RAS, está o acesso à saúde da população migrante. Na esfera contemporânea da migração, confere que os fluxos migratórios internacionais constituem em mudanças sociopolíticas e econômicas, com repercussões globais e locais, constantemente acionadas e aprofundadas sob o domínio do processo da globalização. Essas repercussões acarretam um grande desafio para a saúde pública brasileira, em termos de abrangência e de acesso a essa população. 
Sabe-se que os imigrantes em geral são mais vulneráveis social e economicamente, sobretudo os recém-chegados, que se submetem mais facilmente a postos de trabalho insalubres, a salários baixos e a moradias precárias. Esses aspectos favorecem ao maior risco de quadros de subnutrição, doenças laborais e doenças infecciosas como tuberculose, hepatites e HIV, além de transtornos psicossociais decorrentes das mudanças sociais e do afastamento da família, como depressão, alcoolismo e consumo de drogas (CARBALLO e NERUKAR, 2001; RAMOS, 2009).

Segundo os dados estatísticos fornecidos pelo Ministério da Justiça e Segurança Pública (2019) o relatório anual de 2019, revela que de 2010 a 2018 foram registrados no Brasil 774,2 mil migrantes, considerando todos os amparos legais. Desse total, destacamse 395,1 imigrantes de longo termo (cujo tempo de residência é superior a um ano), composto principalmente por pessoas oriundas do hemisfério sul (CAVALCANTI et al., 2019). Torna-se perceptível que a migração seja um ponto a ser discutido, em especial, por apresentar segundo as estatísticas a migração de populações de países em extrema pobreza, subdesenvolvidos ou desenvolvidos, como a China, porém onde atualmente sofre com problemas de superpopulação. Em termos de Brasil e de saúde pública, isso, pode apresentar certas barreiras em questão de excessiva demanda, o que impacta diretamente na economia e, com isso, na qualidade do serviço prestado, tanto pela rede de serviços como pelos profissionais de saúde.

Inseridos na integração ao acesso à saúde à população de migrantes, encontramse os profissionais de saúde, estes presentes na APS e demais serviços de saúde. Na APS, os profissionais de saúde são direcionados para a Estratégia de Saúde da Família (ESF), que compõem o grupo de profissionais: médico da família; enfermeiro; técnico de enfermagem; agente comunitário de saúde (ACS); dentista e auxiliar administrativo (PNAB, 2017). Para relembrar, a APS é a porta de entrada para demais serviços de saúde, os profissionais são os responsáveis por garantir o acesso à saúde da população, o que inclui os migrantes.

Com isso, o estudo apresenta a problemática: Como é compreendido o processo de acesso à saúde realizado pelos profissionais de saúde ao migrante contemporâneo? Para responder a esse questionamento, estruturou-se o objetivo geral: analisar as concepções dos profissionais da saúde acerca do acesso à saúde prestado à população migrante. 


\section{METODOLOGIA DE ESTUDO}

Estudo de abordagem qualitativa, exploratório-descritiva. Optou-se em desenvolver em etapas qualitativas, com base em Minayo (2014), pois a proposta dialética é considerada como a que melhor responde às necessidades da pesquisa social no campo da saúde. Nessa perspectiva, o projeto de pesquisa insere-se na vertente qualitativa. Segundo a autora, a abordagem qualitativa busca responder questões muito particulares, valorizando os significados, aspirações, motivos, valores, atitudes e crenças, o que corresponde a um espaço mais profundo das relações. Neste aspecto, a interpretação dos fenômenos e a atribuição de significados são básicas no processo investigativo, que busca compreender em profundidade o fenômeno estudado no cenário em que ocorre e do qual faz parte (MINAYO, 2014).

A porção do território em análise é aqui representada pelos municípios de Igrejinha, Rolante e Taquara pertencentes a região do Vale do Paranhana, que corresponde a uma microrregião do Estado do Rio Grande do Sul e tem como setor econômico de maior representatividade a indústria calçadista, predominantemente exportadora. O setor vem sendo afetado gradualmente por crises econômicas que tornaram as médias e grandes indústrias da região menos competitivas, devido à concorrência de indústrias mundiais. Este território, no entanto, ainda é reconhecido pela produção de calçados.

No que se refere às migrações, a concentração de indústrias pode representar um forte atrativo da força de trabalho, evidenciando que essa região se tornou uma área de atração e retenção de migrantes. Os estudos mais recentes sobre a economia regional destacam que a dinâmica socioeconômica do território estaria dependente da interação das firmas e dos agentes sociais de uma forma não apenas mercantil, mas de relações econômicas intangíveis (BRANDÃO; COSTA; ALVES, 2006, BRANDÃO, 2011).

Portanto, neste primeiro estudo na região do Vale do Paranhana, foram escolhidos o profissional enfermeiro e o ACS. O enfermeiro atua como coordenador da equipe de saúde na (APS) e o ACS, por ser um profissional que atua diretamente com a comunidade, através das visitas domiciliárias (VD). Para a realização do estudo, foram entrevistados 7 enfermeiros e 2 ACS, com duração de 15 a 20 minutos. O período de coleta de dados foi nos meses de outubro a novembro de 2019. 
Os dados foram analisados através da análise de conteúdo temática, proposta por Minayo (2014), a qual consiste em agrupar as unidades de significação em núcleos de sentido, os quais constituem uma comunicação em que a frequência, a presença ou mesmo a ausência, possuam algum significado para o objeto de estudo. Através disso, a análise temática propõe três etapas para o método analítico: pré-análise, exploração do material e tratamento dos resultados e interpretação.

Este estudo segue as orientações da resolução 466/12 do CNS/CONEP (BRASIL, 2012) referente às pesquisas com seres humanos, assim como também a resolução 510/16 do CNS/CONEP (BRASIL, 2016) sobre a Ética na Pesquisa na área de Ciências Humanas e Sociais. E se apresenta com apreciação aprovada, através do parecer: 3.554.938 CAAE: 02040318.1.0000.8135. Para manter o anonimato dos entrevistados, foram utilizados as siglas (Enf), para os enfermeiros, seguido do numeral, sendo (Enf-1; Enf-2;...Enf-7) e para os ACS, empregou-se a sigla, seguido do numeral, a exemplo (ACS-1 e ACS-2)

Este estudo é uma série de um projeto guarda-chuva, onde prevê mais etapas de estudo, como; entrevistas com os usuários migrantes e grupos focais com os demais profissionais que não foram incluídos neste presente momento. Os pesquisadores ressaltam que devido a pandemia da COVID-19, algumas dessas etapas foram adiadas e terão retorno previsto para o ano de 2022.

\section{RESULTADOS E DISCUSSÃO}

Através das análises elucidou em três categorias temáticas: "como cheguei aqui? ” o processo de inserção e acesso à saúde dos migrantes; barreiras para o atendimento prestado aos migrantes residentes; e significados que permeiam os profissionais de saúde no atendimento ao migrante, as quais elucidam os principais aspectos encontrados pelas entrevistadas dos profissionais.

\section{“Como cheguei aqui?” O processo de inserção e acesso à saúde dos migrantes}

Conforme relatado pelos entrevistados, para a inserção do migrante no Sistema Único de Saúde (SUS), deve-se realizar o cadastramento, através de documentos que comprovam a sua residência no município. Cada município possui a sua especificidade no cadastramento, no município de Igrejinha, o cadastramento é realizado na própria 
Unidade de Saúde, porém, nos dois municípios restantes (Taquara e Rolante) esse procedimento é realizado pela secretaria de saúde. Após o cadastramento em todos os municípios, é solicitado que o ACS realize uma visita domiciliar para que o migrante tenha acesso a consulta clínica, assim, integrando a Unidade de Saúde.

Eles têm o CPF e o comprovante de residência, que eles nos trazem e então pedimos para o ACS [Agente Comunitário de Saúde] ir lá, que é da área deles, ver se eles realmente moram ali e a partir dali se faz o cadastro com a confirmação da visita do Agente Comunitário de Saúde. Depois da confirmação eles fazem o cartão SUS na própria Unidade. (Enf-1)

O que nós cobramos deles é um comprovante de endereço, se caso não tem pedimos um comprovante no cartório que comprove a moradia, após realizar o cadastramento do SUS [Sistema Único de Saúde] na secretaria de saúde. (Enf-3)

O cadastramento é feito com todos os habitantes do município, independentemente de sua origem. Não tendo o cadastro, mesmo os não migrantes não poderão consultar na ESF [Estratégia de Saúde da Família] do seu bairro - poderão utilizar apenas o hospital. O cadastro ocorre por meio de visita do agente comunitário de saúde. (ACS-1)

Nas falas citadas acima, nota-se que a realização do Cartão Nacional do Sistema Único de Saúde (CNS), se faz imprescindível para o indivíduo ter acesso nos serviços disponibilizados, dentro da unidade de estratégia de Saúde da Família, documento este solicitado pelos profissionais aos Migrantes em sua primeira busca ao serviço. Para o Ministério de Saúde (BRASIL, 2020) o Conselho Nacional de Saúde (CNS) é um documento que promove uma base de dados, de informações geradas durantes atendimentos dos cidadãos em todo o território nacional, promovendo um acesso universal, intersetorial e multidisciplinar das informações e histórico de saúde/doença de cada pessoa atendida dentro do território, independentemente de sua nacionalidade.

Conforme a PNAB (2017), das atuações dos agentes comunitários de saúde que devem ser desenvolvidas na APS, concentra-se diversos papéis fundamentais e importantes, para o funcionamento e o desenvolvimento da promoção de saúde e prevenção de doenças e agravos, em um determinado território, sendo esse, o principal objetivo da atuação da equipe de estratégia de Saúde da Família.

Das atribuições do ACS, destaca-se o cadastramento dos indivíduos e suas famílias, como, o acompanhamento através de visitas domiciliares periódicas, para cadastrados residentes em sua microárea de atuação. Nas visitas domiciliares realizadas 
pelo ACS, busca-se informações importantes para o desenvolvimento de ações e estratégias em Saúde executadas pelas equipes de Saúde da Família (BRASIL, 2017).

A visita domiciliária é feita pelo ACS [Agente Comunitário de Saúde] uma vez por mês e quando vem uma família nova, normalmente o usuário/migrante vem aqui e após é realizado a VD [Visita Domiciliária] e o cadastramento para que eles ficam vinculados com a Unidade. (Enf-1)

Todos os moradores do município são acompanhados pelos agentes de saúde dos seus bairros. A organização não se dá em função dos migrantes, mas sim em função do número total de famílias cadastradas pelos agentes, as quais devem ser visitadas mensalmente. (ACS-1)

Nas discussões do campo de pesquisa, por exemplo, no caso as visitas às oficinas de confecção e residências de imigrantes, estão coladas às ações em Atenção Primária à Saúde (conhecida também como Atenção Básica à Saúde) são desenvolvidas dentro da rotina de visitas realizadas pelo ACS, inseridos na Estratégia Saúde da Família (ESF) (GOLDBERG, SILVEIRA, 2013; SILVEIRA et al, 2016).

Os agentes comunitários de saúde, tem seu trabalho vinculado diretamente com uma equipe de ESF, que é composta por uma equipe multidisciplinar (Médico generalista ou especialista em saúde da família, enfermeiro generalista ou especialista em saúde da família, técnico de enfermagem e agentes comunitário de saúde, podendo ser acrescentado à essa composição um cirurgião dentista e auxiliar de saúde bucal).

Para atuação dessas equipes, recomenda-se um número máximo de 4.000 pessoas cadastradas, podendo esse número ser menor, conforme o grau de vulnerabilidade das famílias do território a ser abrangido pela equipe. No entanto, o número de ACS deve ser suficiente para cobrir $100 \%$ dos indivíduos e famílias cadastradas nesta equipe, não ultrapassando o número máximo de 750 pessoas por agente comunitário de saúde, conforme informações disponibilizadas pela Política Nacional de Atenção Básica (PNAB) (BRASIL, 2017).

Aqui temos o ACS [Agente Comunitário de Saúde], porém tem [áreas] descobertas, então se eles estão nessas áreas somente em casos de necessidade de visita ou acompanhamento. (Enf-4)

Até o momento ainda não realizei visita, mas a área que ele reside está coberta por mim, a questão do horário que implica essa realização, porque não temos na Unidade o horário do trabalhador. E já foi pedido pela gestão para não realizar VD [Visita Domiciliária] fora do nosso horário de trabalho. (ACS-2) 
Temos acompanhamento dos ACS [Agente Comunitário de Saúde] nas áreas cobertas, porém atualmente estamos com 6 das 12 microáreas descobertas por ACS [Agente Comunitário de Saúde], então as visitas seriam programadas conforme necessidade do usuário, e não de forma programática. (Enf-2)

Conforme as falas representadas acima, nota-se que equipes incompletas e/ou horários de funcionamento das unidades, bem como à não possibilidade de o ACS realizar as visitas domiciliares em horários distintos; dificulta no trabalho e acompanhamento dos agentes comunitários de saúde para seus cadastrados, sendo eles migrantes ou não. Essa dificuldade ou falta de visitas periódicas aos usuários e famílias, para Andrade et al (2017), corrobora para um enfraquecimento das ações de promoção em saúde desenvolvidas pelas equipes de estratégia de saúde da família e estreitando o vínculo entre usuário e serviço de saúde.

\section{Barreiras para o atendimento prestado aos migrantes residentes}

Uma vez que a comunicação verbal, entre o profissional de saúde e o usuário, pode estar diretamente relacionada à qualidade da assistência prestada, em uma unidade de saúde, além de, através da comunicação que se estabelece o vínculo entre usuário estabelecimento de saúde - profissional de saúde (CHUBACI, et al 2002). Portanto, nos relatos é perceptível que os profissionais que atuam nas ESFs, acabam por enfrentar essa barreira no atendimento com o migrante.

Um dos desafios é a linguagem, o idioma mesmo. Porque eles chegam aqui sem falar muito o nosso idioma. (Enf-3)

O que nos marca mais é quando eles vêm buscar atendimento e não conseguem se comunicar com eles, então a gente não sabe se está conseguindo ajudar de fato ou não, o problema maior que temos é a questão da língua e comunicação. (Enf-1)

A principal dificuldade é a comunicação; muitas vezes eles custam a entender o que está sendo explicado em função do idioma. (ACS-1)

Contudo, nota-se que apesar do idioma demonstrar ser um dificultador, no processo de interação para o cuidado entre profissionais e migrantes, o esforço por parte de ambos para uma interação assertiva no processo de saúde, acaba gerando estratégias de comunicação. Uma das estratégias utilizadas pelo entrevistado, foi integrar um familiar 
próximo do migrante, o qual era mais fluente na língua portuguesa para repassar as informações sobre a consulta, como segue no relato abaixo:

$\mathrm{O}$ atendimento a uma gestante que chegou aqui na cidade já grávida e iniciou o pré-natal aqui conosco, a questão da comunicação, ela não compreendia o nosso idioma. Então em todas as consultas, o marido estava presente para traduzir a consulta, mas aos poucos ela começou entender o que a gente conversava com ela, mas ainda com muita dificuldade. (Enf-4)

Conforme Ramos, et al (2011), a interação entre prestadores de serviços de saúde e usuários, se qualifica muitas vezes por uma comunicação não verbal, onde possibilita que se imprima sentimentos, emoções e qualidade na fala. Assim, permite o indivíduo não somente perceber e compreender o que as palavras significam, mas criando um ambiente, onde até mesmo o silêncio pode transmitir inúmeras mensagens em um determinado contexto. Por isso, com relação ao atendimento ao migrante é importante que o profissional utilize como estratégia de comunicação, a comunicação não-verbal durante as consultas ou visitas domiciliares.

Para além da comunicação, vale ressaltar que o sistema público de atenção à saúde no Brasil, tem suas dinâmicas de oferta, bem como, as portas de entrada do acesso à saúde, programas de prevenção de doenças/agravos, programa específico de imunizações, prénatal e puerpério. O que, por vezes, acaba criando uma realidade distante da vivência do país de origem do migrante, podendo assim, trazer dificuldade no entendimento da dinâmica de assistência ao indivíduo que vive no território brasileiro (GUERRA e VENTURA, 2017).

[...] Outra coisa, que notamos que eles não trazem as crianças com tanta regularidade, o que causa atraso de vacinas, isso muito também pela questão de acesso, transporte, muitos deles não tem carro, o que é mais visto são as bicicletas, então isso dificulta mais essa questão. (Enf-1)

É na verdade eles procuram mais a Unidade quando estão doentes, eles não vêm como os brasileiros que sabem a rotina de agendamento, para fazer exame de rotina, ver como está a saúde do migrante não, eles só procuram quando estão adoecidos. (Enf-1)

Não procuram muito a unidade, somente gestantes ou crianças, relutam em relação a vacinação. (Enf-6)

Sim, são mais as mulheres que buscam o serviço de saúde, para consultas com o ginecologista, em todas as consultas os maridos acompanham as esposas, pela questão cultural mesmo [...]. (Enf-6) 
Os migrantes muitas vezes ressignificam ou mudam suas concepções e práticas sobre o processo saúde-doença nos serviços de assistência em saúde do seu novo território, porém, utilizam com menor frequência os serviços públicos de saúde, quando comparados aos "pertencentes da terra". (SILVEIRA et al., 2016). A falta de conhecimento/instrução quanto a forma de se pensar e promover saúde no território brasileiro, relacionado com questões culturais adquiridas em seu país de origem, acaba por manifestar-se em hábitos de cuidado, no qual pode parecer impróprio na percepção do profissional de saúde brasileiro, como é perceptível nas falas a seguir:

[...] Até mesmo tive um caso agora que falei para a usuária que ela deveria ficar 15 dias sem intercurso sexual, devido a um aborto que ela teve, e ela me respondeu com uma risadinha, ou seja, a questão sexual para eles fica muito a critério do homem e não da mulher. (Enf-6)

Ano passado tivemos uma haitiana que teve um problema cardíaco e fiquei sabendo que ela foi até o Haiti para fazer um tratamento e depois ela voltou, então até ficamos sem entender... será que ela não confiou no diagnóstico e tratamento daqui? (Enf-1)

A forma como é visto o cuidado em saúde e o "choque cultural", entre usuário migrante e equipes do sistema público de saúde brasileiro, acaba corroborando para um cenário que requer sensibilidade no atendimento por parte do profissional de saúde. Sendo esse, um momento crucial para a criação de vínculo e representação de um local de acolhimento e confiança para o migrante (MARTIN et al, 2018).

\section{Significados que permeiam os profissionais de saúde no atendimento ao migrante}

O profissional enfermeiro, que se encontra a frente da gestão e organização do processo de trabalho nas equipes de saúde da família, bem como, o profissional responsável junto a equipe de saúde no acompanhamento do trabalho do ACS (BRASIL, 2017). Tem como premissa de sua profissão o cuidado em saúde, nesse contexto, faz-se pensar entre a relação do profissional e o usuário, que quando tratado dessa forma, gera um sentimento que deve ser levado em consideração durante a assistência prestada a qualquer indivíduo.

Com esse pensamento, os profissionais de saúde envolvidos no cuidado e assistência da população migrante, acabam por sentir-se gratos, pelo fato de usarem os 
seus conhecimentos técnicos/científicos para acolher e proporcionar uma experiência positiva e criação de vínculo com esses migrantes de forma que contribua para uma assistência de qualidade.

Me sinto muito agradecido e valorizado por estar fazendo algo útil a quem precisa, pois eles demonstram gratidão e carinho por estarem recebendo um tipo de atenção pública que não tinham em seu país de origem. (ACS-1)

Me sinto empolgado, para conhecer outra cultura ou visão de forma de viver. (Enf-2)

Feliz em saber que nosso sistema é totalmente gratuito e que estamos recebendo estrangeiros. Me orgulho em dizer que não deixamos ninguém sem atendimento, enquanto em alguns países isso ocorre. (Enf-6)

Me sinto um pouco frustrada por nem sempre entenderem as orientações, penso que deveria ter políticas públicas para melhor acolher os imigrantes, por exemplo aulas de português. (Enf-5)

A gente se sente bem despreparado, porque muitas vezes a gente não consegue entender o que eles querem e nem saber se estamos fornecendo o que eles precisam [...] (Enf-1)

Com esse pensar, a Política Nacional de Humanização, Brasil (2003), conhecida também, como, Humaniza-SUS do Ministério da Saúde, foi desenvolvida para reforçar a importância da assistência em saúde humanizada, sem discriminação e garantindo a integralidade e universalidade, que está entre os princípios do SUS. Os trabalhadores do sistema de saúde, quando apropriado dessa política e princípios, acabam por desenvolver uma assistência mais humana, buscando compreender a subjetividade do ser humano, acolhendo-o de forma integral e adequada para suas necessidades.

A partir de uma conquista histórica brasileira, dada com a Constituição de 1988, que visa a universalidade do acesso à saúde, se fez um direito de todo cidadão em território nacional, sem haver distinção de classe, cor, etnia, especificidade de gênero etc., sendo ele de nacionalidade estrangeira ou nacional (SANTOS, 2016). Os profissionais ainda encontram dificuldades organizacionais nos serviços de saúde, que podem interferir na assistência prestada ao migrante, nas falas a seguir podemos perceber que eles demonstram desejos e pensam em estratégias de enfrentamento para essas barreiras.

Acredito que seria importante que eles fossem mais bem acolhidos e instruídos no Município, porque eles chegam aqui sem nenhum 
conhecimento da nossa língua, então isso acaba sendo uma grande barreira para nós. (Enf-6)

Como a maior barreira que temos é a comunicação, até pensamos em uma reunião em algum projeto com a Assistência Social para ensinar o português para eles. (Enf-1)

Acredito que deveria se ter um horário do trabalhador para que eu pudesse realizar as visitas domiciliárias tanto para o migrante quanto para o usuário trabalhador, pois isso prejudica um pouco a minha cobertura e acompanhamento de área. (ACS-2)

Elenca-se que os profissionais que se encontram na linha frente do atendimento em saúde ao imigrante (nesse estudo, enfermeiro e ACS), identificam que o idioma é um dos maiores dificultadores ao acesso dessas pessoas, e que apesar da universalidade ser uma das premissas do SUS, esse acesso pode acabar sendo falho.

Tanto para os profissionais de saúde que estão diretamente ligados a assistência da população de migrantes, como, para os próprios migrantes, a mídia se faz uma ferramenta importante, pois através dela que será possível a inserção do migrante no contexto do novo ambiente, principalmente quando marcado por preconceito de raça e classe (SILVA, 2017). Com essa visão, as redes podem contribuir para divulgação de ações de cuidado, saúde e integração do migrante para com a sua comunidade e aproximação dos serviços disponíveis na rede para a assistência integral do indivíduo.

Eu acho que a gente sente não só aqui, mas que não temos muito conhecimento sobre esses migrantes, mas como funciona, não lemos muito e até a mídia não divulga também. (Enf-4)

Temos alguns haitianos que residem na área de atuação do ESF, mas não tenho conhecimento se existe no município algum programa para receber migrantes. (Enf-5)

A escassez de programas e políticas públicas voltadas para a assistência e inserção da população migrante no contexto social, acarreta um déficit que pode acabar manifestando no seu próprio autocuidado, na ausência de assistência e/ou negligência com os acompanhamentos de saúde, como, consultas médicas ou ações de saúde. Sendo que o profissional, que está na ponta da rede de atenção em saúde para a prestação de serviços, acaba ficando a mercê de estratégias do próprio local de trabalho, para suprir uma necessidade de gestão e/ou política pública vigente e efetiva para o cuidado com o migrante (BAENINGER et al, 2017). 
Os relatos dos profissionais, como a Enf-1 e o ACS-1, ressalta a percepção de um povo acolhedor e ao mesmo tempo conservador, por vezes, preso às suas origens e culturas, mas que compreende a necessidade de inserção na comunidade. A interação e laços, que se criam entre os profissionais de saúde e a população migrante, garante uma assistência de qualidade e o estabelecimento de vínculos fortificados.

\footnotetext{
O que chama a atenção é que entre eles [haitianos], são muito unidos e nos tratam bem, é uma cultura diferente... por exemplo, quando faço visita domiciliar eles são bem acolhedores, nos atendem bem. (Enf-1)

Os migrantes [haitianos] são muito gratos e honestos, e isso resplandece no sorriso com que eles normalmente nos recebem; são trabalhadores e muito unidos. (ACS-1)
}

Para Miranda (2018) o Brasil por ser um país com fortes raízes com o fenômeno migratório, vem mostrando nas últimas décadas, um papel de forte relevância no cenário mundial dos fluxos migratórios internacionais. A inserção desses fluxos, no território nacional, acaba corroborando para um contexto de maior aceitação e interação da comunidade, criando por vezes estratégias próprias para somar com esse migrante no novo território. Com isso, é vista a necessidade de estruturar estratégias para o recebimento dos migrantes dentro das comunidades e da atenção primária à saúde, assim como, na capacitação dos profissionais para o atendimento desses migrantes.

Contudo fica iminente que os profissionais de saúde enfrentam alguns dilemas em relação ao atendimento e acesso à saúde desses migrantes, em grande parte pela questão da comunicação, relacionado ao idioma, o que dificulta a transferência de orientações relacionadas à saúde e ademais está relacionado a diversidade cultural que o migrante integra as suas práticas de cuidado. Dias et al (2011) ressalta que a capacitação dos profissionais de saúde para lidarem com esta diversidade pode ser um componente importante da formação dos recursos humanos, contribuindo para uma maior adequação dos serviços às necessidades das populações imigrantes e para uma maior equidade em saúde.

\section{CONSIDERAÇÕES FINAIS}

Atualmente, o Brasil se encontra em um contexto político, econômico e social extremamente delicado, porém, a exemplo de estratégias de saúde e profissionais 
empenhados, para melhoria do atendimento ao usuário estrangeiro, inserindo-o em seu sistema público de saúde. O SUS acolhe e atende as pessoas que o procuram, sem nenhuma distinção, que se adapta a pluralidade cultural deste território, de modo que o sistema seja efetivamente universal e integral.

Na visão dos profissionais de saúde, embora existam barreiras que podem acabar distanciando o migrante dos serviços de saúde, como; idioma e as diferentes culturas de promoção de saúde e prevenção de doenças, é uma população que deve ser muito bem trabalhada para a sua inserção nos serviços de saúde. A importância de estabelecer um vínculo entre profissional de saúde e migrante se faz imprevisível para que o acesso à saúde aconteça, o profissional pode se empoderar da estratégia de comunicação nãoverbal, quando a comunicação verbal se encontra ineficiente. Outro aspecto é estabelecer o vínculo efetivo, entre profissional - serviço de saúde - usuário migrante, sendo possível desta forma trabalhar questões relacionadas à prevenção e promoção à saúde, tais, são objetivos principais da atenção primária à saúde.

Reconhecer as diferenças culturais de cada população é de extrema importância para os profissionais de saúde, através disso, o profissional terá mais facilidade em se inserir no cuidado em saúde do migrante. Não se pode esquecer, da busca pelo respeito mútuo entre as partes, que tem como finalidade, o profissional de saúde validar conhecimento adquirido do usuário anteriormente no seu país de origem, assim, estabelecer novas estratégias de cuidado em conjunto. O SUS é um sistema de grande abrangência, e garante o acesso universal a todos que estão inseridos no território brasileiro, deve-se reconhecer as barreiras, que tornam os serviços de saúde frágeis no acesso a população migrante, e através desse reconhecimento traçar estratégias para as estreitar os desafios, tanto por parte da comunicação como da informação oferecida a esses migrantes.

\section{REFERÊNCIAS}

ANDRADE, Viviane et al. Visitas Domiciliares de Agentes Comunitários de Saúde: Concepções de Profissionais e Usuários. Psico-USF, Bragança Paulista, v. 22, n. 1, p. 87-98, jan/abr. 2017.

BRANDÃO, C. A. Visões teóricas sobre desenvolvimento regional e a questão das escalas (mundial, nacional, subnacional e local) nas políticas regionais contemporâneas. Curso Territorialidade e Políticas Públicas no Brasil. Brasília: ENAP, v. 2, 2011. 
BRANDÃO, C.; COSTA, E. J. M.; ALVES, M. A. S. Construir o espaço supranacional de articulação socioprodutiva e das estratégias de desenvolvimento: os novos arranjos institucionais. In: DINIZ, Clélio Campolina e CROCCO, Marco. Economia Regional e Urbana - contribuições recentes. Belo Horizonte: UFMG, p. 196-224, 2006.

BRASIL, Ministério da Saúde. Cartão Nacional de Saúde, 2020. Disponível em: https://www.saude.gov.br/saude-de-a-z/doacao-de-sangue/693-acoes-eprogramas/40031-cartao-nacional-de-saude. Acesso em: 20/04/2021.

BRASIL. Sistema Único de Saúde (SUS): estrutura, princípios e como funciona. Brasília, 2019.

BRASIL. Política Nacional de Atenção Básica - PNAB. Brasília, 2017.

BAENINGER, R.; PERES, R. Migração de crise: a migração haitiana para o Brasil. R. bras. Est. Pop., Belo Horizonte, v.34, n.1, p.119-143, jan./abr, 2017.

CARBALLO, M; NERUKAR, A. Migration, refugees, and health risks. Emerging Infectious Diseases, v.7, n.3, p.556-560. 2001.

CAVALCANTI, L. et al. A inserção do imigrante, solicitante de refúgio e refugiado no mercado de trabalho formal. Observatório das Migrações Internacionais; Ministério da Justiça e Segurança pública/Conselho Nacional de Imigração e Coordenação Geral de Imigração Laboral. Brasília, DF. 2019.

CHUBACI, R. et al. A comunicação no processo da hospitalização do imigrante. Rev Latino-am Enfermagem; v. 10, n. 6, p. 805-12, 2002.

DIAS, S. et al. Barreiras no acesso e utilização dos serviços de saúde pelos imigrantes. Acta Medica Portuguesa, v. 24, n. 4, 2011.

GOLDBERG, A.; SILVEIRA, C. Social inequality, access conditions to public health care and processes of care in bolivian immigrants in Buenos Aires and São Paulo: a comparative inquiry. Saúde e Sociedade, v. 22, n. 2, 2013.

GUERRA, K.; VENTURA, M. Bioética, imigração e assistência à saúde: tensões e convergências sobre o direito humano à saúde no Brasil na integração regional dos países. Cad. Saúde Colet., v. 25, n. 1, p. 123-129, Rio de Janeiro, 2017.

MARTIN, D. et al. Immigration, refuge and health: sociocultural analysis in perspective. Saúde Soc. São Paulo, v. 27, n.1, p.26-36, 2018.

MENDES, E. V. As redes de atenção à saúde. 2. ed. Brasília: Organização PanAmericana da Saúde, 2011.

MINAYO, M. C. S. O desafio do conhecimento: pesquisa qualitativa em saúde. ed. 14. São Paulo: Hucitec, 2014. 
MIRANDA, S. C. A História em espiral: compreendendo a receptividade brasileira à imigração haitiana a partir de suas determinações. Aedos, Porto Alegre, v. 10, n. 22, p. 29-52, Ago. 2018

RAMOS, N. Saúde, migração e direitos humanos. Mudanças: Psicologia da Saúde, v. 17, n.1, p.1-11. 2009.

RAMOS, A.; BORTAGARAI, F. A Comunicação não-verbal na área da saúde. Rev. CEFAC, São Paulo. 2011.

SANTOS, F. V. A inclusão dos migrantes internacionais nas políticas do sistema de saúde brasileiro: o caso dos haitianos no Amazonas. História, Ciências, Saúde Manguinhos, v. 23, n. 2, abr.-jun, 2016.

SIDNEI, A. S. Imigração e redes de acolhimento: o caso dos haitianos no Brasil. R. bras. Est. Pop., Belo Horizonte, v. 34, n. 1, p. 99-117, jan/abr, 2017.

SILVEIRA, C. et al. O lugar dos trabalhadores de saúde nas pesquisas sobre processos migratórios internacionais e saúde. Cadernos de Saúde Pública, v. 32, p. e00063916, 2016.

\section{Recebido em: 15/09/2021}

Aprovado em: 05/10/2021

Publicado em: 10/10/2021 